\title{
Erythema neonatorum toxicum
}

Toxic erythema of the newborn was originally described by Bartholomaeus Netlinger in 1472 and renamed erythema neonatorum toxicum by Leiner in $1912 .{ }^{1}$ It is a benign self limited eruption that may be present at birth in up to $20 \%$ of cases, ${ }^{2}$ although more typically it occurs 24 to 48 hours after birth. ${ }^{3}$ Roughly $30-70 \%$ of newborn infants are affected. There is no racial or sexual predilection but there is increased frequency of erythema toxicum in infants with increasing birth weight and gestational age. ${ }^{4}$

Clinically, the lesions are firm, 1-3 $\mathrm{mm}$, pale yellow or white papules and/or pustules on a red and swollen base. The resultant picture is that of a 'flea bite'. Atypically, the lesions may occur as single red macules, confluent macules, or irregular splotchy erythema up to $3 \mathrm{~cm}$ in size. There is a predilection for the lesions to occupy the anterior and posterior trunk but lesions may readily be found on the upper arms, thighs, and face. The palms and soles are almost never involved. Erythema toxicum usually lasts about five to seven days as crops of new lesions that heal without residual pigmentation. The eruption does not usually recur, though several questionable episodes have been documented within the month. No systemic involvement has been noted, though serum eosinophilia is seen in $7-18 \%$ of cases and tends to correlate positively with severity of the disease. ${ }^{5} \mathrm{~A}$ diagnosis can be made routinely by preparation of a Tzanck smear, Giemsa stain, or Wright stain preparation of a pustule, which will show numerous eosinophils. (The Tsanck smear consists of removing the blister roof and preparing a slide from the scrapings of the base of the blister using a scalpel blade.) Histologically, the pustules are most often located in the epidermis just beneath the stratum corneum and are filled with eosinophils. Histological examination of a macule usually shows a perivascular infiltrate composed primarily of eosinophils and a few polymorphonuclear leucocytes and mononuclear cells.

The aetiology of erythema toxicum remains a mystery, though several hypotheses have been put forth. Keitel et al considered the atopic diathesis as an antecedent cause but found that the incidence of atopy in the families of affected infants was not greater than that in the general population. ${ }^{4}$ The possibility that erythema toxicum was the result of an immediate hypersensitivity reaction to an allergen has been entertained, but no specific antigens or antibodies have ever been implicated. ${ }^{2}$
A superficially more plausible hypothesis is concerned with the fragility of newborn skin. As erythema may readily be induced in newborn skin with light pressure and vesiculation by brief rubbing it might be suspected that erythema toxicum may be related to mechanical or thermal stimulation. ${ }^{4}$ This hypothesis, however, does not explain why all newborns do not have erythema nor does it take into account the greater fragility of premature skin and the propensity of erythema toxicum to await full gestation before presenting itself as a problem to the infant. The production of eosinophilic pustules may be related more to a peculiar reaction of the skin of predisposed individual's experience than to a specific cause. If erythema toxicum is a reaction pattern of the skin we have yet to understand how to separate this particularly predisposed subpopulation. The aetiology of erythema toxicum is still unknown at present and there is no treatment needed because eruption resolves spontaneously and it has no known serious complications.

\section{Differential diagnoses}

Transient neonatal pustular melanosis. Transient neonatal pustular melanosis is more often present at birth than erythema toxicum and occurs more often in mature black neonates of both sexes. ${ }^{6}$ There is no sexual preference for the disorder. Erythema toxicum and transient neonatal pustular melanosis can be seen together in the same infant and the lesions of each disorder readily separated morphologically. Transient neonatal pustular melanosis initially starts as very superficial vesiculopustular lesions. When the lesions decompress or burst they leave pigmented macules with a surrounding collerette scale. The lesions are most often present under the chin, on the forehead, the nape, the lower back, and occasionally the trunk, extremities, the cheeks. ${ }^{7}$ Absent is the 'flea bitten' appearance of erythema toxicum, representing the peripustular oedema. The eruption may last several weeks to a few months. Histologically, the vesiculopustule is usually located beneath the stratum corneum and is filled with polymorphonuclear leucocytes or eosinophils, or both. Presence at birth, different clinical presentation, and a typical histologic picture help, therefore, to differentiate the two diseases.

Eosinophilic pustular folliculitis. Eosinophilic pustu- 
lar folliculitis was originally described by Ofuji. ${ }^{8}$ It is characterised by recurrent crops of follicular papulopustules located on the face, trunk, and extremities. Histologically, an intense infiltration of the hair follicle with eosinophils is seen. Lucky et al have recently described a number of such patients, one of whom was a newborn who was thought to have severe erythema toxicum. ${ }^{9}$ The recurrent nature of the disorder, its predilection for the scalp, and associated peripheral eosinphilia noted during the attacks helped, however, to differentiate the two diseases.

Incontinentia pigmenti. Incontinentia pigmenti (Bloch-Sulzberger disease) is an $\mathrm{X}$ linked, dominantly inherited disease characterised by three dermatological stages. Initially, linear vesicular lesions that are usually present at birth evolve into verrucous lesions within weeks and are then followed by a peculiar swirled hyperpigmentation that may last for years. The vesicular and keratotic stages may each last from months to years. They are most often located on the trunk and extremities. Multisystem involvement is common with evidence of neurological, skeletal, ocular, and dental abnormalities occurring as the patient ages. ${ }^{10}$ Histologically, a biopsy specimen of a vesicule would show a subcorneal or intraepidermal pustule filled with eosinophils. The histology is identical to erythema toxicum but the persistence of the eruption, the evaluation through different stages, and multiple system involvement help to differentiate the two diseases.

Infantile acropustolosis. Infantile acropustolosis was originally described by Kahn ${ }^{11}$ and by Jarratt ${ }^{12}$ and is characterised by recurrent crops of 1-2 mm intensely pruritic vesiculopustules found previously on the distal extremities of infants. It is more common in black infants and is predominantly found between the ages of 2 and 10 months. Several reports exist of onset in the neonatal period. ${ }^{13}$ Histologically, subcorneal or intraepidermal pustules that are filled with polymorphonuclear and eosinophilic leucocytes and a sparse perivascular infiltrate may be found. Several cases with eosinophil filled pustules have been noted. ${ }^{14}$ The characteristic distribution, recurrent nature, and predominant polymorphonuclear cell component in the pustules, however, set this order apart from erythema toxicum.

Other disorders. Several other diseases bear men- tion in this discussion of neonatal vesiculopustular eruptions. Miliaria is an extremely common eruption in the neonate secondary to sweat retention and is characterised by a vesicular eruption with subsequent maceration and obstruction of the exocrine ducts. The lesions are most often seen in the intertriginous areas, particularly the neck and axillae. Vesiculopustular eruptions of infectious aetiology, such as herpes simplex, varicella, or cytomegalovirus, must also be ruled out with a Tzanck smear preparation and culture, impetigo with routine Gram stain and culture, candidiasis with routine potassium hydroxide preparation and culture, and scabies with appropriate mineral oil preparations. Letterer-Siwe disease is characterised by the presence of purpura mixed among the eczematous papules, which contain the atypical histiocytes characteristic of this disorder.

\section{References}

1 Carr JA, Hodgman JE, Freedman RI, et al. Relationship between toxic erythema and infant maturity. Am J Dis Child 1966;112:129-34.

2 Levy HL, Cochran F. Erythema toxicum neonatum present at birth. Am J Dis Child 1962:103:617.

${ }^{3}$ Solomon LM, Esterly N. Neonatal dermatology. Philadelphia: W B Saunders, 1973.

${ }^{4}$ Keitel H, Yadov V. Etiology of toxic erythema. Am J Dis Child 1963;106:306-9.

5 Moschella SL. Hypersensitivity and miscellaneous inflammatory disorders. In: Moschella SL, Pillsbury DM, Hurley HJ, eds. Dermatology, Vol 1. Philadelphia: W B Saunders, 1975:396-7.

6 Wyre HW Jr, Murphy MO. Transient neonatal pustular melanosis. Arch Dermatol 1979;115:458.

7 Ramamurthy RS, Reveri M, Esterly NB, et al. Transient neonatal pustular melanosis. $J$ Pediatr 1976;88:831-5.

* Ofuji S, Ogino A, Horio T, et al. Eosinophilic pustular folliculitis. Acta Derm Venereol (Stockh) 1970;50:195-203.

${ }^{9}$ Lucky A, Esterly N, Heskel N, Krafchik B, Solomon LM. Eosinophilic pustular folliculitis in infancy. Pediatric Dermatology 1984;1:202-6.

10 Wiklund D. Incontinentia pigmenti. Arch Dermatol 1980; 116:701

${ }^{11}$ Kahn G. Acropustulosis of infancy. Arch Dermatol 1979; 115:831-3.

12 Jarratt M, Ramsdell W. Infantile acropustulosis. Arch Dermatol 1979;115:834-6.

13 Jennings JL. Infantile acropustulosis. J Am Acad Dermatol 1983;9:733.

${ }^{14}$ Bundino S. Infantile acropustulosis. Dermatologica 1982; 165:615-9.

FAnNie J Berg and Lawrence M Solomon Department of Dermatology, University of Illinois,

Chicago,

Illinois 60680,

United States of America 